\title{
Langue et « patrimoine » immatériel
}

Portrait de l'espace géographique et anthropique

\section{Federica Diémoz}

\section{(2) OpenEdition}

\section{Journals}

Édition électronique

URL : http://journals.openedition.org/edl/492

DOI : $10.4000 /$ edl. 492

ISSN : 2296-5084

Éditeur

Université de Lausanne

Édition imprimée

Date de publication : 15 mai 2013

Pagination : 143-166

ISBN : 978-2-940331-32-1

ISSN : 0014-2026

Référence électronique

Federica Diémoz, «Langue et « patrimoine » immatériel », Études de lettres [En ligne], 1-2 | 2013, mis en ligne le 15 mai 2016, consulté le 19 décembre 2020. URL : http://journals.openedition.org/edl/492 ; DOI : https://doi.org/10.4000/edl.492 


\section{LANGUE ET «PATRIMOINE» IMMATÉRIEL. PORTRAIT DE L'ESPACE GÉOGRAPHIQUE ET ANTHROPIQUE}

Historiquement, la plus grande partie de la Suisse romande, à l'exception du Jura, appartient au domaine linguistique du francoprovençal, langue qui est encore utilisée par la dernière génération de locuteurs fribourgeois (gruériens) et valaisans. Les données de l'Atlas linguistique audiovisuel du francoprovençal valaisan permettent d'observer de quelle manière les dialectes francoprovençaux organisent la description de la réalité topographique alpine, en particulier dans le domaine des expressions spatiales. Ces parlers reflètent également un patrimoine immatériel des connaissances qui témoignent, en Suisse romande comme ailleurs, d'un ancrage très fort au territoire. Un riche corpus de contes, légendes et récits inédits en francoprovençal valaisan illustrera bien l'attachement des habitants à l'espace géographique et anthropique.

«Nous habitons tous quelque part, mais la localisation n'est jamais neutre, dans aucune société."

M. Segaud, Anthropologie de l'espace, p. 71

\section{Introduction}

«L'espace est une société de lieux-dits, comme les personnes sont des points de repère au sein du groupe.»

C. Lévi-Strauss, La pensée sauvage, p. 202 sq.

Dans le domaine des études linguistiques, les rapports entre l'espace et la langue, entre l'espace vécu et les personnes qui non seulement occupent tel ou tel lieu mais qui se l'approprient, renvoient naturellement aux désignations des noms de lieux. L'onomastique et plus particulièrement 
la toponymie illustrent au mieux les dynamiques qui régissent le rapport entre la langue et l'espace. Comme le souligne Fabre, «le corpus des désignations toponymiques d'une commune n'est pas autre chose que la construction même du paysage par la communauté linguistique des lieux " ${ }^{1}$. La toponymie fournit un portrait assez fidèle de la vision de l'homme et du paysage que chaque communauté a élaborée au cours de son histoire. Les noms de lieux témoignent aussi bien des activités économiques, agricoles, sociales d'un lieu, que des changements du paysage liés à la flore ou à la faune qui caractérisent un lieu précis. Ils constituent par ailleurs un dépôt de la mémoire collective des habitants qui véhicule des croyances, des habitudes, des pratiques s'inscrivant dans un territoire donné. Les études toponymiques contribuent ainsi à conserver l'identité historique et culturelle d'une communauté et fournissent de riches informations accessibles aux historiens, aux géographes, aux linguistes, aux archéologues.

En Suisse romande, des travaux systématiques de récolte sur le terrain des noms de lieux, entrepris au début du $\mathrm{XX}^{\mathrm{e}}$ siècle par les fondateurs du Glossaire des Patois de la Suisse Romande (GPSR), en particulier par Ernest Muret, constituent une importante documentation qui est encore en cours d'exploitation ${ }^{2}$. Il s'agit d'une vaste enquête sur les noms de lieux dans presque mille localités de la Suisse romande ainsi que le dépouillement des plans cadastraux. Plus récemment, le Dictionnaire toponymique des communes suisses ${ }^{3}$ documente les origines linguistiques de la Suisse à travers le témoignage des noms de lieux.

Si ces travaux toponymiques constituent un axe de recherche incontournable, j'aimerais toutefois mettre ici en relief d'autres éléments linguistiques qui fonctionnent également comme clefs de lecture du territoire. Dans cette contribution, je vais ainsi aborder un aspect qui a très peu - ou presque jamais - été étudié, à savoir le domaine des expressions spatiales dans les parlers francoprovençaux valaisans. Il s'agit d'une étude qui ne se veut pas exhaustive mais qui permettra toutefois de voir émerger certaines tendances significatives.

I. P. Fabre, «Ce que la toponymie peut apporter à la ... toponymie», p. 13.

2. Le GPSR est un ouvrage lexicographique fondamental qui documente le patrimoine lingustique et culturel de la Suisse romande. Depuis 1899, il apparaît sous forme de fascicules et illustre la richesse et la variétes des parlers historiques de cette région.

3. A. Kristol et al., Dictionnaire toponymique des communes suisses. 
Les expressions spatiales dans les parlers francoprovençaux

Si la richesse phonétique et lexicale des parlers francoprovençaux (pl. I.1) a déjà fait l'objet d'importants travaux dialectaux - en particulier les Tableaux phonétiques des patois suisses romands, le GPSR et de nombreuses monographies dialectales -, il reste beaucoup à faire dans le domaine de la morphosyntaxe de l'oralité dialectale. C'est dans ce cadre que, depuis 1994, un atlas de nouvelle génération est en cours d'élaboration au Centre de dialectologie de l'Université de Neuchâtel. Il s'agit de l'Atlas linguistique audiovisuel du francoprovençal valaisan (ALAVAL), axé principalement sur des questions de morphosyntaxe et basé sur une importante documentation vidéo et sonore. Constituée de 25 points d'enquête (21 localités en Valais, deux en Vallée d'Aoste et deux en Haute-Savoie), il rassemble environ 15’000 clips audio-visuels dans lesquels des locuteurs natifs reformulent librement des énoncés français qui leur étaient soumis.

Pour ce qui est de la deixis spatiale, il est connu que les langues romanes et les langues germaniques expriment de manière très différente les concepts spatiaux. Les langues telles que l'allemand ont tendance à exprimer le déplacement au moyen d'une particule (adverbe ou préfixe) en position sœur du verbe, tandis que pour les langues littéraires standardisées d'origine latine, telles que le français ou l'italien, on a tendance à penser qu'elles préfèrent exprimer le déplacement par un verbe simple: en français "sortir", "entrer", "monter» en italien uscire, entrare, salire, scendere. En réalité, il s'agit là d'une manière de dire qui a été imposée à la communauté linguistique dans le cadre de l'établissement de la norme littéraire, au XVII e siècle en ce qui concerne le français.

Dans ses travaux sur le romanche, Berthele ${ }^{4}$ a bien mis en évidence le comportement de cette langue romane qui est dans une situation de contact avec l'allemand. D'après son analyse, il en ressort que les trois variétés des parlers romanches étudiés (vallader, surmiran et sursilvan) ont un fonctionnement plus semblable aux parlers germaniques qu'aux langues romanes, à savoir un usage restreint des verbes de déplacement compensé par des adverbes de déplacement et des prépositions. On peut pourtant se demander si l'adstrat germanique a vraiment influencé les

4. R. Berthele, "Contact de langues et conceptualisations spatiales». 
parlers romanches ou s'il a plutôt consolidé une tendance qui existe depuis toujours dans la syntaxe romane populaire ${ }^{5}$.

Il devient ainsi intéressant de voir de quelle manière se comportent les parlers francoprovençaux, langues galloromanes qui se situent dans une aire-carrefour entre les domaines linguistiques gallo-italique et gallo-roman, langues qui ont été tantôt définies de transition ${ }^{6}$ et tantôt - probablement à tort - même influencées par les langues germaniques ${ }^{7}$ :

Demandez à un Valaisan de quel nom il appelle en son patois un lieu familier et comment il a coutume de dire qu'on y est ou qu'on y va: souvent, dans sa réponse, avant la préposition attendue, surgira un adverbe, indiquant chaque fois la direction qu'a suivie la pensée pour localiser une présence, un état, une activité, ou pour accompagner dans son trajet un corps en mouvement. Le même usage se constate dans les textes valaisans mis au jour par MM. Jeanjaquet et Fankhauser dans les Légendes de Savièse récemment publiées par l'abbé Luyet [... $]^{8}$.

D’après Muret, ces parlers alpins préfèrent utiliser des adverbes et des prépositions joints aux verbes, plutôt que des verbes de déplacement.

Dans les matériaux $A L A V A L$, pour reformuler l'énoncé français «Pour monter sur le toit, je prends une échelle", dix témoins ont utilisé la forme du verbe "aller» seule et douze ont préféré utiliser le verbe «aller» suivi de l'adverbe «en haut». Les locuteurs d'Evolène et de Bionaz disent:

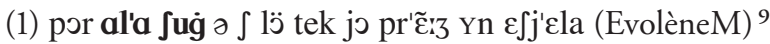
Pour aller en haut sur le toit je prends une échelle.

(2) p al'i si 6 lo t'ẹtœ mə f'a pъ'çndy 1 its'rila (BionazM)

Pour aller en haut sur le toit il me faut prendre l'échelle.

Un seul témoin, celui de Torgnon en Vallée d'Aoste, a utilisé le verbe «monter» doublé de la préposition "par-dessus»:

5. W. N. Mair, «Transferenz oder autonome Bildung?».

6. R. Sornicola, «Per una tipologia del parlato nelle lingue romanze», p. 65 sq.

7. G. Tuaillon, «Le francoprovençal s'explique-t-il par les Burgondes?».

8. E. Muret, "Adverbes préposés à un complément de lieu dans les patois valaisans», p. 79.

9. Les matériaux de l'ALAVAL sont transcrits en API, suivis par une traduction littérale française. Entre parenthèses le nom de la localité où s'est déroulée l'enquête, ainsi que la lettre $\mathrm{M}$ ou $\mathrm{F}$ pour indiquer le sexe de l'informateur. 
(3) p'є̨

Pour monter dessus le toit... il me faut prendre l'échelle.

A la différence des parlers romanches étudiés par Berthele ${ }^{10}$, les parlers valaisans connaissent également des verbes de déplacement tels que mountà (ou l'équivalent lexical pojì dans les parlers valdôtains), souvent suivis par un ou deux adverbes: "monter en haut" ("descendre en bas»), "monter en amont» ("descendre en aval»), "monter en amont en haut» ("descendre en aval en bas»), "monter en haut plus haut» («descendre en bas au dessous») qui sont utilisés couramment par les locuteurs.

Le témoin d'Evolène, en décrivant la dalle qui empêche les souris de monter dans le raccard ${ }^{11}$, dit:

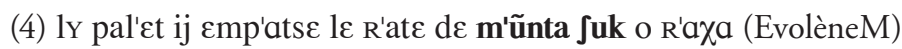

La dalle empêche les souris de monter en haut au raccard.

Sur le même modèle, suite à la question ouverte: «Est-ce qu'il y a une montagne qu'on voit bien dans la vallée?", l'informatrice de Torgnon explique spontanément que pour voir le Mont Cervin, il faut:

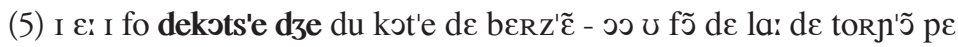

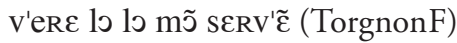

Eh il faut descendre en bas du côté de Berzin... au au fond de la de Torgnon pour voir le le Mont Cervin.

Dans les parlers francoprovençaux valdôtains ${ }^{12}$, des tournures telles que bèichì ba dezó "descendre en bas au-dessous" ou pujì si dameun «monter en haut au-dessus» sont tout à fait courantes et d'usage quotidien.

Ce besoin de préciser les indications spatiales, se retrouve également avec des syntagmes tels que "sortir dehors» - «entrer dedans":

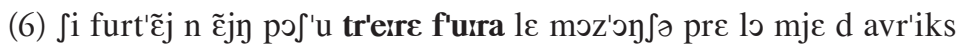
(EvoleneM)

Ce printemps nous avons pu sortir dehors les génissons vers la mi-avril.

Io. R. Berthele, «Contact de langues et conceptualisations spatiales».

II. L'énoncé demandé aux témoins est: "La dalle empêche les souris de monter dans le raccard.»

I2. Le parler francoprovençal de Roisan, en Vallée d'Aoste, est ma langue première. 


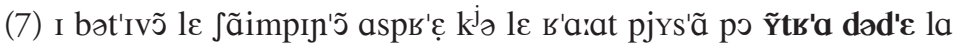
gs'ãdzə (TorgnonM)

Ils mettaient les champignons (dalles) exprès que les souris ne puissent pas entrer dedans la grange.

Pour exprimer un déplacement, les langues romanes peuvent, par ailleurs, joindre aux verbes "aller", "venir", "courir", "monter", "entrer", "sortir» et autres semblables un adverbe de direction qui en précise ou en renforce la signification: en italien andar via, montar su, scender giù, entrar dentro, uscire fuori, etc.

Ce qui distingue les parlers valaisans et valdôtains de leurs voisins, c'est la fréquence avec laquelle ils associent les mêmes ou d'autres adverbes à un complément de lieu dans les phrases dont le verbe n'exprime pas un déplacement: "travailler en bas", "être en haut", "dormir en bas». Pour l'énoncé du questionnaire $A L A V A L$ «Tu travailles au jardin ", 39 témoins ont ajouté l'adverbe «bas», alors que 11 ont employé la forme verbale seule. Le témoin masculin de Montana dit ainsi :

(8) tø trav'a $\alpha \varepsilon \varphi$ ba ว kurt' $\varnothing$ (MontanaM)

Tu travailles en bas au jardin.

Dans l'énoncé «Ma voisine est née à Sierre», notre informatrice d'Arbaz précise spontanément que Sierre se trouve "en haut", dans la vallée du Rhône ${ }^{13}$, alors que dans la phrase "Nous achetons les clous à la quincaillerie en ville», le témoin de Bionaz ajoute «en bas» pour se référer à la ville:

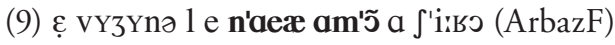

Ma voisine est née en haut à Sierre.

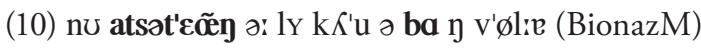

Nous achetons euh les clous euh en bas en ville.

Les exemples ci-dessus, parmi les nombreux cas relevés dans les données de l'ALAVAL, attestent que la configuration topographie du territoire valaisan et valdôtain, constitué par les plaines du Rhône et de la Doire, avec leurs vallées latérales, semble rendre l'axe vertical essentiel dans

I3. En réalité, Arbaz est situé à une altitude de 1146 mètres, alors que Sierre, dans la plaine du Rhône, se trouve à 533 mètres... «En haut» ne désigne donc pas toujours l'altitude absolue, mais, dans ce cas précis, la position relative au cours du Rhône. 
l'expression spatiale et non seulement avec des verbes de déplacement. Par ailleurs, l'exemple $n^{\circ} 9$ montre que l'adverbe «en haut» ne désigne pas toujours l'altitude absolue; une analyse approfondie des riches matériaux $A L A V A L$ - qui ne peut pas être développée ici - pourrait illustrer les différentes significations de cet adverbe.

Les expressions spatiales dans la pratique du contage, dans la "littérature orale»

La nécessité de la part des Valaisans de préciser leur pensée, de localiser un acte, se manifeste également dans l'acte de la narration, dans la pratique du contage.

E. Muret, «Adverbes préposés à un complément de lieu dans les patois valaisans", p. 79.

Dans le cadre d'un projet Sinergia ${ }^{14}$, axé sur le Patrimoine immatériel, nous travaillons actuellement sur un important corpus de manuscrits reflétant la tradition orale de deux régions linguistiques différentes de la Suisse romande: le Jura et le Valais. Il s'agit de contes et récits inédits recueillis sur le terrain au cours du $\mathrm{XX}^{\mathrm{e}}$ siècle lors de veillées et de rencontres informelles, situations ancrées dans la réalité quotidienne d'autrefois. Comme l'a bien démontré Simonsen ${ }^{15}$, la pratique du «contage» a presque disparu en Europe, hormis pour les enfants. Il est regrettable que les folkloristes se soient intéressés prioritairement au contenu des contes et non aux conditions de réalisation de la narration orale (en tant que forme esthétique originale et phénomène anthropologique). Il existe d'ailleurs une abondante littérature de tradition orale destinée au grand public et beaucoup de contes recueillis par des

I4. Cette recherche constitue le sous-projet B («Le conte traditionnel en Suisse romande: de l'oral à l'écrit et vice-versa») du projet FNS-Sinergia "Intangible Cultural Heritage: the Midas Touch" - sous la direction de la prof. Ellen Hertz - et parrainé par l'Institut d'ethnologie de l'Université de Neuchâtel, en collaboration avec les Universités de Bâle (Seminar für Kulturwissenschaft und Europä̈sche Ethnologie), Lausanne (Institut d'anthropologie), le Musée d'ethnographie de la ville de Neuchâtel, le CNRS (Laboratoire d'anthropologie et d'histoire de l'institution de la culture, Paris), et la Haute Ecole-Arc (Institut horlogerie et création, La Chaux-de-Fonds).

I5. M. Simonsen, Le conte populaire français. 
dialectologues avec des objectifs linguistiques, mais ces matériaux n'ont pratiquement jamais été exploités de manière scientifique et les questions qui nous concernent sont très peu étudiées.

Nos matériaux permettent de documenter encore la pratique naturelle de la transmission orale d'un savoir culturel et ethnographique. Pour les deux sous-corpus à notre disposition, jurassien et valaisan, nous possédons également les informations concernant les pratiques du travail de la collecte, la date, le lieu et l'identité du conteur, détails qui sont souvent absents dans les éditions de contes et récits. Il faut cependant souligner que ces matériaux ne sont pas des ethnotextes ou des transcriptions phonétiques précises, mais des notations écrites de la part des enquêteurs. Le transfert à l'écrit d'une version orale, sans le support d'un enregistrement, est un exercice difficile et exigeant: la tradition écrite exerce inévitablement une influence sur l'enquêteur.

L'étude scientifique de la littérature orale a pris le relais des grandes collectes des folkloristes des XIX ${ }^{\mathrm{e}}$ siècle et début du XX $\mathrm{XX}^{\mathrm{e}}$ siècle. Elle constitue une discipline carrefour ouverte sur des approches interdisciplinaires (littérature, histoire, ethnologie, linguistique, psychologie, psychanalyse, pédagogie, orthophonie) et embrasse une diversité de formes et de genres en perpétuel mélange et renouvellement. Les anthropologues ont beaucoup travaillé sur les contes. Pour eux, étudier la littérature orale est un moyen de comprendre une société, ses idées, ses croyances, ses valeurs et sa manière de négocier les relations sociales dans un espace donné ${ }^{16}$.

Il est nécessaire de distinguer, parmi les genres de cette tradition populaire, un plus ou moins grand degré de formalisation puisque il y avait des textes qui pouvaient être modifiés au cours de la narration et d'autres, par contre, immuables, fixés par des formes métriques ou musicales. Parmi les textes qui peuvent être modifiés, on trouve les contes proprement dits qui, à cause de leur complexité et de leur longueur, peuvent à chaque répétition présenter des variantes lexicales ou stylistiques, tout en conservant les motifs et l'ordre de déroulement. La pratique du contage, propre à chaque civilisation humaine, comprend les légendes, les anecdotes et les récits, les contes proprement dits pour les enfants, les contes d'animaux et de magie (dans lesquels apparaissent des éléments surnaturels), avec des thèmes largement répandus en

I6. W. P. Murphy, «Oral Literature». 
Europe, transmis par des conteurs et conteuses professionnels ou non. Il est regrettable qu'une bonne partie de cette documentation ne nous soit pas parvenue dans sa version originale: au début du $\mathrm{XX}^{\mathrm{e}}$ siècle, le folkloriste Arnold Van Gennep a recueilli sur le terrain des centaines de contes en Savoie et dans le Dauphiné, mais ces contes sont publiés en français. Il en est de même des Novelle italiane publiées dans les années 1960 par Italo Calvino, Novelle qui sont des versions italiennes des textes dialectaux provenant de presque toutes les régions italiennes et de la Corse.

Une particularité de notre documentation est donc que nous disposons des transcriptions de la langue orale de contage, ce qui permet d'en étudier certaines particularités linguistiques, phénomènes qui sont souvent absents dans des traductions libres. Des structures telles que "aller en haut", "aller en bas" sont régulièrement traduites par «monter", "descendre». On en trouve des exemples dans les récits La peste à Nendaz ${ }^{17}$ et La Peste à Savièse ${ }^{18}$ mais les auteurs nous fournissent également la transcription phonétique du récit dans les deux variétés francoprovençales valaisannes, ce qui donne l'occasion de relever le fonctionnement linguistique en question.

Le corpus valaisan est constitué d'environ 300 récits francoprovençaux manuscrits et inédits recueillis par Rose-Claire Schüle dans sa monumentale entreprise ethno-linguistique conduite dans la commune de Nendaz depuis $1946^{19}$, ainsi que d'une dizaine de contes manuscrits recueillis dans la même commune en 1906 par Jules Jeanjaquet, conservés aux archives du Glossaire des patois de la Suisse romande (GPSR). Schüle a noté les récits que les gens racontaient spontanément pendant les veillées; à Nendaz, à cette époque, la pratique du contage n'était pas liée à un conteur spécialisé. Ce corpus témoigne encore d'une transmission orale et naturelle d'un savoir culturel et ethnographique.

$\mathrm{Si}$, d'un côté, les contes, légendes, récits véhiculent des motifs connus partout en Europe, ils témoignent de l'autre d'un ancrage très fort au territoire. L'espace géographique est ainsi toujours très bien précisé: la localisation exacte des lieux où se déroule l'action avec une abondante

I7. J. Jeanjaquet, «La peste à Nendaz».

I8. B. Luyet, "Légendes de Savièse», p. 31 sq.

19. R.-C. Schüle, Inventaire lexicologique du parler de Nendaz (Valais); ead., Les vouivres dans le ciel de Nendaz. 
utilisation des déictiques. Il en va de même des personnages qui sont évidemment originaires des lieux de la commune: fées de Brignon, fées de Clèbes, fantôme du glacier du Giétroz, fantôme du Rhône, lac magique de Valais, etc., et nous dévoilent aussi des pratiques et des activités liées au territoire, à l'espace anthropique. Ainsi, le récit de L'homme qui épousa une fée nous apprend qu'un système particulier de maturation du blé était utilisé autrefois à Nendaz, alors que le récit Le fantôme $d u$ Giétroz nous renseigne sur les croyances et les conventions liées à certains espaces communautaires, à savoir les alpages.

\section{L'espace géographique}

Dans de très nombreux récits de l'arc alpin, un personnage plus ou moins mythique récompense un être humain, généralement une femme, pour un service rendu en lui offrant des objets apparemment sans valeur comme une poignée de charbons ou de feuilles. Comme la personne est gratifiée de ce qui lui semble bien inutile, elle ne s'en préoccupe pas lorsqu'en route, la majeure partie des éléments s'échappent de son tablier ou de son récipient. Arrivée à domicile, une particule, restée cachée à l'insu de l'héroïne, se révèle être de l'or. Malgré une recherche entreprise immédiatement, les charbons ou feuilles dispersés auparavant restent introuvables ${ }^{20}$.

Dans le récit de Nendaz (reproduit ici en en alphabet phonétique international, avec les indications du lieu et de la date de l'enquête, avec les initiales du témoin, le tout accompagné d'une traduction littérale) ${ }^{21}$ il s'agit d'une petite fille qui rend service à une fée. Cette dernière la récompense généreusement en lui demandant toutefois de ne pas chercher à savoir quelle est sa récompense avant d'être arrivée chez elle. La curiosité l'emporte et la fille se retrouve avec des objets de peu de valeur

20. Ch. Abry, "La légende des feuilles changées en or à Sixt (Haute-Savoie)", p. 78-85; A. Cérésole, Légendes des Alpes vaudoises, p. 89; G. Isler, "Or ou charbon?», p. 1295.

2I. Les récits ont été transcrits par Schüle en système "Boehmer-Bourciez», ils ont été ensuite translittérés en utilisant le système phonétique international (API). Une deuxième translittération en graphie commune pour les patois valaisans, une graphie simplifiée élaborée par Maître, Pannatier (R. Maître, G. Pannatier, «Graphie commune pour les patois valaisans») est prévue pour rendre ces matériaux accessibles au grand public. 
(des feuilles). Ce récit est encore bien connu par les habitants de Nendaz et, lors d'une enquête de terrain en avril 2011, nous avons pu en enregistrer une version actuelle en francoprovençal. Observons maintenant la présence des expressions spatiales, marquées en gras dans un extrait de ce récit:

Les feuilles changées en or

Haute-Nendaz, H.P. 3 janvier 1948 (fig. 1)

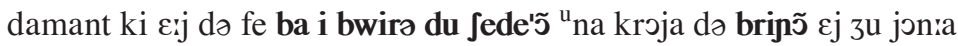
pə ma'rejna. un ku sta fe ir innwe dəmæn'da də 'krãma an ma:r da mat:cta, ma kum jej 'pa paskjə e ats erõy əmu əm plã $\int w \varepsilon$ e kə e tsa'su erõ pa un'ko ba at’ọ o ase. i marr દj a di k arej fe pọr'ta ba a kroja. kã i a'se e $3 u$ ar ${ }^{\mathrm{r}^{\prime}}$ wa a mitu plen a mœstretia e a fe pọr'ta 'ba a kroja. kan zu wida a mœstretia i fe $\varepsilon$ Jurtetii e kãy $\varepsilon$ tọr'naj drı i mœstreța

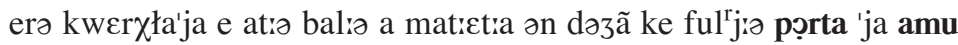

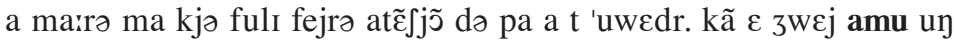

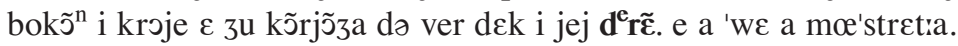
stafi irr plajna də fol: də bjəa. i krojə a mu'za kej pa mãk də pọr'ta

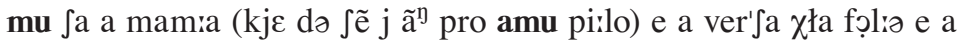
tọr'na a kwerðła a mœstretia.

Du temps où il y avait des fées en bas dans les trous du Chédéon, une fillette de Brignon ${ }^{22}$ en avait une comme marraine. Une fois cette fée était venue demander de la crème à la mère de la fillette. Mais elle n'en avait pas parce que les vaches étaient en haut en baut au mayen à Planchouet et que les hommes n'étaient pas encore en bas avec le lait. La mère a dit qu'elle l'aurait fait porter en bas par la fillette. Quand le lait est arrivé, elle en a rempli une mestrette ${ }^{23}$ et l'a fait apporter en bas par la fillette. Quand la fée a eu vidé le récipient, elle est sortie et quand elle est rentrée dedans la mestrette avait le couvercle fermé. Elle l'a donnée à la fillette en lui disant de porter cela en haut à sa mère mais qu'elle devait faire attention de ne pas l'ouvrir. Lorsque la petite a été un bout plus haut, elle a été curieuse de voir ce qu'il y avait dedans. Elle a ouvert la mestrette. Elle était pleine de feuilles de bouleau. La fillette a pensé qu'il n'y avait aucun besoin de rapporter en haut cela à sa mère, qu'en

22. Hameau qui se trouve dans le haut de la commune de Nendaz.

23. La mestrette est un récipient en bois qui a une douve plus haute que les autres servant de poignée. 
the. Nendaz,

$$
\text { H.P. (M.M.) B. I.48 }
$$

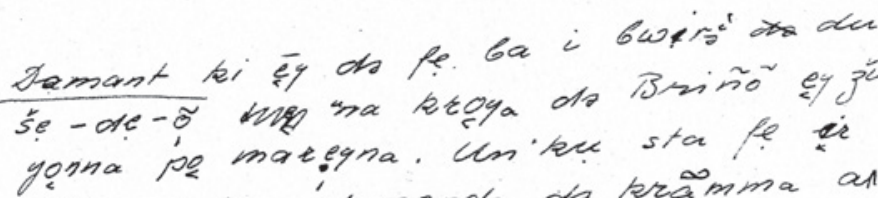

inñwe domanda ds terámma al màr da imattetta, ma bum yey pa paskyo e ats erón amu m Dláswe e ko e trasu enó pa unke ba attoo axe. I mair ey a di t'aréy fe axpporta ba a kequa. Kã i ase egua ariv́a a mitu pley a möste etto e á pe porta ba a breya. Kan z"u wida a möstrętio ifle e surteth e kãy $e$ formay dré i mostretta ers ruẹifteya e atto balla a mattetta in dazía lee fubly pottay $y_{1} y_{1}$ a mu a mōro ma kyo Lubl" feyro atésỹ do pa a t unede. Kầ $e$ jwel a mu un $V$ botaín i kroye e zü koryogia do ver do to dele i yey dré. i a ve a möstretta. Sta su irs pleayna ds fóll do bypa. $\checkmark$ kroy a muźa key pa mấ do posta mu sa a mammax C a versa Zía folls e a toma, a \& kweetea a möstretta. $\times$ (bye dese y ẩ pre a mu pilo)

Fig. 1 - Manuscrit de Rose-Claire Schüle. 
baut, à la maison, il y en avait assez. Elle a renversé le récipient et a remis le couvercle à la mestrette.

Si les noms de lieux servent à bien situer la localisation du récit, les expressions spatiales liées à la verticalité - en haut et en bas - dominent.

Dans le récit de La peste à Nendaz de 1500, on peut également relever l'abondance de détails concernant les noms de lieux ainsi qu'une présence substantielle des adverbes "en haut», "en bas». Dans ce récit, en particulier, on relèvera le besoin de localiser exactement le personnage principal au sein de la commune de Nendaz:

La peste à Nendaz

C.B., février 1967 (fig. 2)

kã a 3y i 'pesta a 'nẽ $\tilde{n}^{\mathrm{n}} \mathrm{da}$, i pa rẽykj a 'nẽ $\tilde{n}^{\mathrm{n}} \mathrm{da}, \partial^{\mathrm{n}}$ va'i, paskj i va'i a i'ta

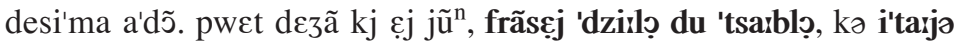

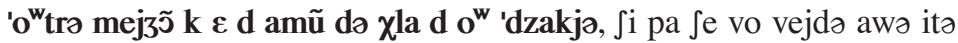

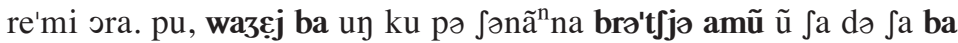

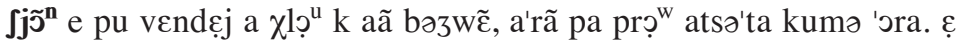
'pэ:ə, kã i'nє̨j amy Ja 'rota, 'irə pa unko i 'rota, dəzo bri'j̃̃ e dəzo a

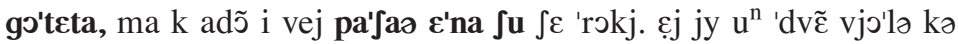

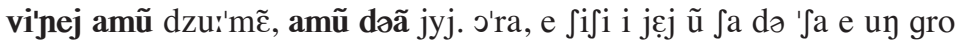
tsa'a k 'irə mun'ta, 'irə mun'ta $\int u$, e fazej prow tsa. kã $\varepsilon$ 3y də 'ku:tə

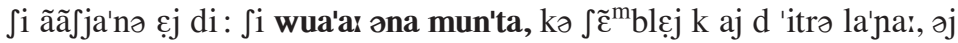

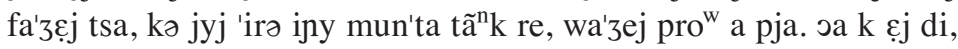

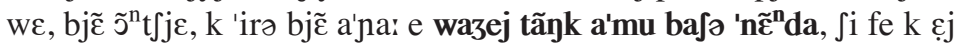
unkọ 'una 'bona 'trota a 'fejrə. a'prej kã $\varepsilon$ zy əna $\int u$ tsa'a ã disku'rej, ke

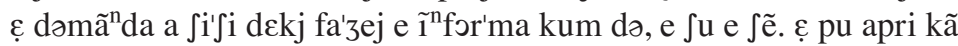

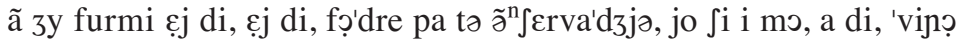

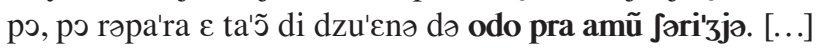

Quand il y a eu la peste à Nendaz, pas seulement à Nendaz, en Valais, parce que le Valais a été décimé alors. Puis ils disaient qu'il y avait un, François Gilloz du Châble, qui habitait en là [dans] la maison qui est en amont de celle du Jacques, je ne sais pas si vous voyez où habite Rémi maintenant. Puis il allait en bas une fois par semaine apporter en haut un sac de sel, en bas à Sion et puis il vendait à ceux qui en avaient besoin, ils en avaient pas assez acheté comme maintenant. Et puis, quand il venait en haut sur la route, il était [il n'y avait] pas encore la route, au-dessous de Brignon et au-dessous de Gotette, mais alors le 
C. Bounban

fécuis 1867

kã a jül ipésta a Nénda, ipa rêry a Neinda, on Vai, pasky' Ho' a ifa' desimó ado'. Preet d' deján, ky'ey yiurn, Frâsey Bzillo du Tsablo, ko' itaýlo owha meysoró k'e d'amũ do yed dón Sjakya, si pa, s'e vo uegda awo it Rem' gira. 'Pu, itiñey, wayé 6a, un ku, pà sonánna bristoy amert ut sta do sta $6 a$ sygn e pu vendey a Ylow klã

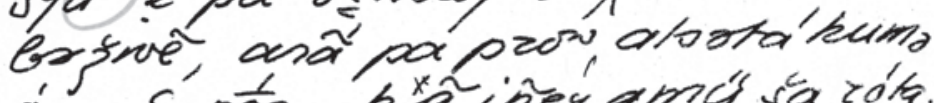

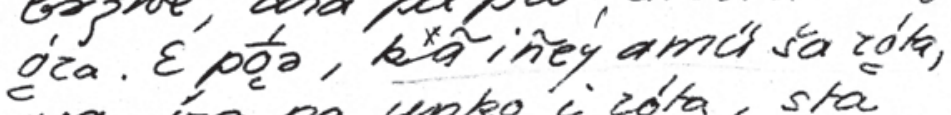
wa, in pa unter i zota, sta dero por cey, dojo Buiñó $e$. diso a goteta, mase rodo'c vey pasás erral se reky. Ey, ges un doé vyoés ka vinéy Orá, e sisisi i yey th s'a do sa 'e un gro saá hy'in munta', iss muntá sul, e foriey puov

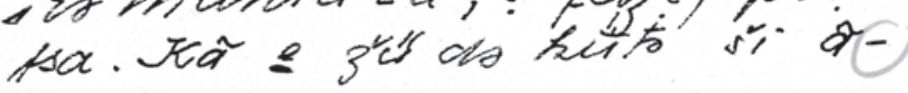


chemin passait en haut sur ce rocher. Il vu un petit vieux qui venait en haut avec beaucoup de peine, en haut devant lui. Alors, celui-ci avait un sac de sel et un gros cheval qui était monté, sur lequel était monté, et il faisait assez chand. Quand il eut [füt] à côté de ce vieux, lui dit s'il voulait en haut monter, qu'il lui semblait d'être fatigué, il faisait chaud, que lui était venu à cheval jusque là, il allait bien à pied. Oh qu'il dit, oui, bien content, qu'il était bien fatigué et il allait jusqu'en haut à Basse Nendaz, ce qui fait qu'il y a encore un bon bout à faire. Après, quand il eu [était] en haut sur le cheval ils ont discuté, qu'il demanda a celui-ci qu'est-ce qu'il faisait et l'informa de quoi, de ci et ça. Et puis, quand ils ont eu fini lui dit, lui dit, il ne faudrait pas t'effrayer, moi je suis la mort, il a dit, je viens pour, pour raccommoder les talons [des souliers] des jeunes de Odo Praz, en haut à Cerisier. [...].

Dans le début d'un autre récit portant sur le crapaud, on trouve:

Jean-Pierre Bourban était un homme très dévotieux. Tous les jours le matin, avant d'aller en haut [travailler] à la forêt, il allait communier en bas à Basse-Nendaz (D.B., 18 octobre 1966).

Dans le territoire valaisan, où le relief est la caractéristique principale de la configuration de l'espace géographique, l'opposition haut-bas, en amont-en aval semble être omniprésente.

L'espace anthropique

A Nendaz, c'est la moisson précoce qui sert de base à un récit menant au fier baiser. Ce récit, portant sur l'union entre une fée (évidemment de Brignon, hameau de Nendaz) et un homme de l'endroit illustre l'attachement de l'homme à son territoire. Lunion entre les deux se passe bien, ils ont deux filles, mais elle se termine mal au bout de quelques années, à cause d'une promesse non tenue. Le mari avait en effet dû promettre de ne jamais prononcer la formule fawa arâdze, charvâdze, mais un jour, il se met en colère et prononce les mots fatidiques parce que la fée - qui avait prévu des intempéries - a rentré la moisson avant maturité en mettant entre le blé des branches de verne (aulne). La fée disparaît, mais il reste une possibilité de pardon: la fée se transforme en serpent que le mari doit embrasser. Toutefois, celui-ci n'en a pas le courage et la fée disparaît. 
La fée qui épousa un humain

H.P(M.M) Hte Nendaz 3.1.48

jej un ku un ma'trõ k ej ma'rja una fe di kretro də briñ̃. ma njun Jaã kj ern una fe paskjə sta fi દj jej di kj fọ'li war'da o sək'rœ. a bu də kakjə

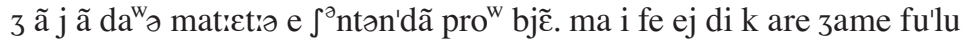

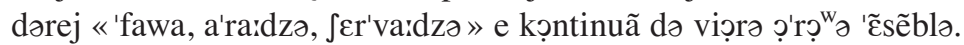
un ku i jyj દ̣rə partej po di trẹj dzo. e i 'fenna bjẽ kə i bla unko zu fẽ

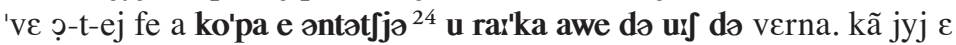
zu tọr'na a ba'rak:a e k a ju $\int \tilde{\varepsilon} \int \varepsilon t$ əngrenza ej ja kə'rja «'fawa, a'ra:dzə,

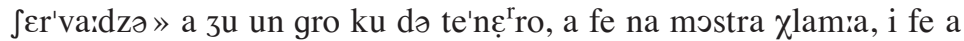
disparu. dəktrə dz ${ }^{\mathrm{w}}$ aprej a $3 \mathrm{u}$ də məstrə 3 ग़'radzo, awə də plədzi e

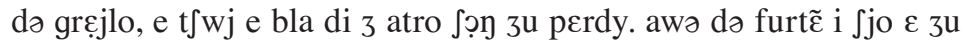
bว̃ mu o ra:'ka. i fe vine t $\int w \varepsilon$ dzo kã jyj iirə virə pə pi'nə e 'kroje, e

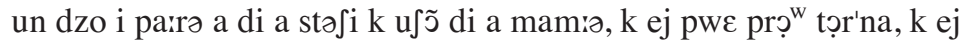

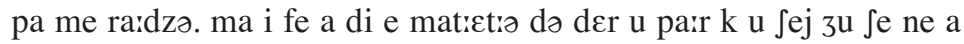

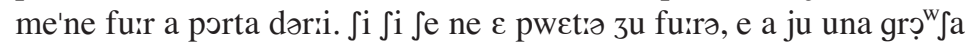

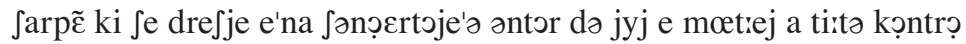

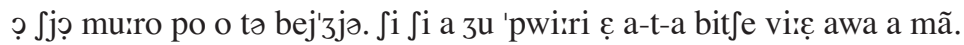

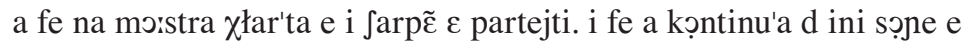
mat:عt:ə, ma jyj a-t-a zame plu ju'fa.

Il y avait une fois un homme qui avait épousé une fée des Crêtes de Brignon. Mais personne savait qu'elle était une fée parce que celle-ci avait dit qu'il fallait garder le secret. Au bout de quelques années ils ont eu deux filles et ils s'entendaient très bien. Mais la fée lui dit qu'il n'aurait jamais fallu lui dire "fawa arâdze, chervâdze" et ils continuaient de vivre heureux ensemble. Une fois, lui [le mari] était parti pour deux trois jours. La fermme, bien que le blé ềt été encore fin vert, le coupa et l'entassa au raccard avec des branches de verne [aulne]. Quand lui [son mari] il a été rentré à la maison et qu'il a vu ça, il s'est fâché et il a crié "fawa arâdze, charvâdze". Il y a eu un gros coup de tonnerre, il a fait une monstre flamme, la fée disparut. Quelques jours après, il y a eu de monstres orages avec de la pluie et de la grêle et tous les blés des autres ont été détruits. Avec le printemps le sien [de blé] était bien mûr au raccard. La fée venait tous les jours quand lui était loin, pour s'occuper des petites et un jour le père a dit à celles-ci qu'elles eussent dit à la maman qu'elle

24. En francoprovençal, l'action d'entasser est exprimée par différents verbes selon la matière: entasser le blé se dit euntitchì alors qu'entasser le bois se dit euntsailli (F. Diémoz, G. Pannatier, «Richesse et variété des patois autour des Alpes», p. 10). 
pouvait bien rentrer, qu'il n'était plus fâché. Mais la fée a dit aux filles de dire au père qu'il ait été cette nuit dehors derrière la porte. Celui-ci il a bien été dehors et il a vu un gros serpent qui se dressait en haut et s'enroulait autour de lui et mettait sa tête contre son visage. Celui-ci a eu peur et il a éloigné la bête avec la main. Il y a eu une grande clarté et le serpent est parti. La fée continua de venir soigner les filles mais lui ne l'a plus jamais vue.

Dans le corpus de Nendaz, on trouve des variantes de ce récit: c'est la localisation interne à la commune qui change (Crêtes de Brignon, hameau de Clèbes); le couple a deux filles ou deux garçons; la rencontre entre le serpent et le mari a lieu derrière la porte d'entrée ou derrière la porte de la cave; la fée vient soigner les enfants ou elle disparaît pour toujours; l'homme n'ose même pas s'approcher du serpent ou fait une tentative de l'embrasser mais l'éloigne de la main. Ce récit est également connu dans d'autres communes du Valais, dans les Alpes vaudoises, et dans le Jura ${ }^{25}$. Ces variantes témoignent bien la créativité individuelle des conteurs et de leur attachement à leur territoire. Dans la transmission de la «littérature» orale, les narrateurs opèrent une sélection personnelle, ils se projettent dans le récit, mettent en œuvre leur capacité d'adaptation, d'ancrage à leur espace, à leur réalité, en répondant à la demande du public.

Pour ce qui est du détail concernant la verne (mot du français régional qui désigne l'aulne), dans les versions de Nendaz, la plupart de narrateurs ont effectivement spécifié qu'il s'agissait de la verne, tandis qu’ailleurs on se contente de mentionner des branchettes, ou même de passer sous silence la méthode adoptée pour faire mûrir le blé.

Un deuxième récit, Le fantôme du Giétroz, du glacier du Giétroz, nous renseigne qu'autrefois, après la désalpe (la descente des troupeaux en plaine), les alpages apparemment inhabités devenaient inacessibles. Seule une raison importante pouvait justifier une montée à l'alpage. Ce récit nous fournit des indications sur l'organisation de l'espace au sein d'une communauté, sur l'interdiction de certains déplacements et sur le respect ou non de ces conventions. Dans l'extrait ci-dessous, un propriétaire doit remonter à l'alpage chercher sa vache, et fera ainsi la rencontre avec des

25. A. Bourdin, Hérémence; H. Correvon, «La Fée de Cleibe»; J. Jeanjaquet, "La fée de Premploz»; L. de Lavallaz, Essai sur le patois d'Hérémence (Valais-Suisse); E. Montelle, W. Richard, B. Brüsch, Les plus beaux contes de Suisse. 
messagers (employés d'alpage) qui seront cordiaux avec lui, alors qu'ils seront méchants avec un homme qui ne monte que pour curioser. Le fantôme du Giétroz viendra en aide au malheureux mais il ne pourra pas empêcher sa mort qui survienda un mois après cette montée à l'alpage.

Le fantôme du Giétroz, du glacier du Giétroz

L. M., février 1967

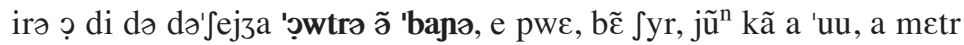

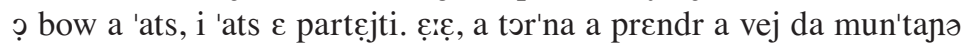

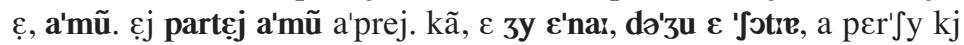

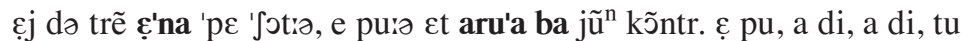

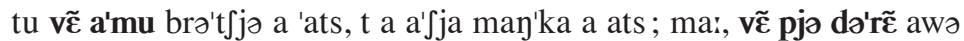

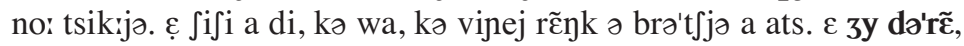
'irõ t t wi, 'irõ na banda də mə'Ja:dzə, 'fazã 'fi:ta. fazã a 'sina, ę ã unkọ

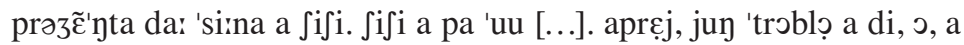
di jo i, i pa 'pwirri pọ a'a ə'na mũ ${ }^{n}$ tanə. a di, wi a'a ə'na jo vera 'kobla

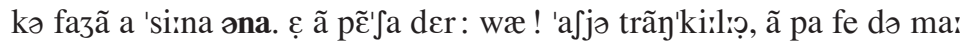

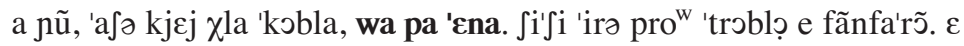
par'tej e'na una ne. $\varepsilon$ pwe ji 'pa:tọ a di: a di, e: bẽ po fejr a ver kə t i 3y ə'na də to, tu prẽn'dre:j o ko ${ }^{w_{1}}$ te, jyj ą̧j a'fja ọ ko ${ }^{w_{1}}$ te k $\mathrm{\varepsilon} m p l e ə$ po $\varepsilon$

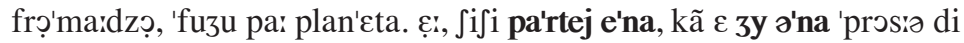

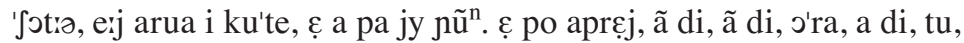

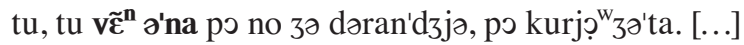

C'était le jour de la désalpe, outre à Bagnes, et puis bien sûr, un [propriétaire] quand a voulu mettre sa vache à l'étable, la vache est partie. Elle était remontée. Il a repris le chemin de la montagne, et, en haut! Il est allé en haut après [la vache]. Quand il est arrivé en haut, sous les chottes [étables d'alpage], il a entendu du trein [bruit] en haut dans les chottes. Et puis est arrivé en bas un à sa rencontre. Il puis a dit, a dit: "Toi, tu viens en haut chercher ta vache, tu as laissé manquer ta vache; mais viens seulement dedans un moment avec nous. "Et celui-ci a dit que oui, qu'il n'était monté que pour chercher la vache. Il est venu dedans, ils étaient tous, ils étaient une bande de messagers [travailleurs à l'alpage], ils faisaient la fête. Ils faisaient le souper et ils ont présenté le souper à celui-ci. Celui-ci n'a pas voulu [...]. Après, un étourdi a dit alors, a dit: "Oh! moi, je n'ai pas peur d'aller en haut à la montagne.» Il a dit, "moi, je veux aller en haut voir moi-même cette bande qui font un souper là-haut!». Les autres ont pensé lui dire: "Ouais! Laisse tranquille, 
ils n'ont fait de mal à personne, laisse cette bande tranquille, ne va pas là-haut!». Celui-ci était un peu fou et fanfaron. Il est parti en baut un soir. Et puis, le fromager de l'alpage a dit, a dit: "Eh bien, pour faire voir que tu as vraiment été là-haut, tu prendras le couteau", il avait laissé le couteau qu'il employait pour le fromage, fiché dans la poutre maîtresse. Et celui-ci est parti en haut, quand il est arrivé en haut près des chottes, il est arrivé au couteau, il n'a vu personne. Et puis après ils lui ont dit, ont dit: "Toi, toi tu viens en haut pour nous déranger, pour curioser. " [...]

\section{Conclusion}

Une première analyse des données $A L A V A L$ et des récits ci-dessus montre que dans les parlers francoprovençaux valaisans et valdôtains, la richesse de l'expression spatiale ne fait pas de doute. C'est en particulier la dimension spatiale de la "verticalité» qui semble jouer un rôle fondamental, dimension reconnue comme première dans l'expérience humaine: «[...] le dimensioni verticali sono infatti più importanti di quelle orizzontali, che possono essere espresse facoltativamente ${ }^{26}$.» Le même auteur forge à ce sujet le titre I sei lati del mondo:

I sei lati del mondo è un'espressione persiana e poi turca usata per cogliere in un colpo solo l'insieme di tutte le localizzazioni spaziali raggiungibili. Molte parole sarebbero necessarie per spiegare quello che è invece del tutto intuitivo: l'orientamento umano individua [...] quattro quadranti dello spazio; e questa prima suddivisione, che già potrebbe bastare ad esaurire lo spazio tridimensionale, è completata dall'opposizione alto-basso. Queste sei direzioni, i sei lati del mondo, sono riassunte in un modelllo tangibile, la tenda dei nomadi, che le sbarra ognuna con un piano - le quattro pareti, il tetto, il piano del suolo - creando quindi per l'uomo un riparo delimitato e circoscritto rispetto alla dispersione nello spazio ${ }^{27}$.

Serait-ce le territoire montagneux du Valais et en particulier son axe vallées/plaine qui a favorisé cet emploi abondant des expressions spatiales? Ce besoin de préciser les indications spatiales semble être

26. G. R. Cardona, I sei lati del mondo, p. 23.

27. Ibid., p. 1. 
plutôt universel, car il apparaît également chez des peuples de la plaine, où le cours d'un fleuve peut fonctionner comme axe de référence ${ }^{28}$.

A quale esigenza esterna risponde dunque un così fine reticolo di indicazioni spaziali obbligatorie? Ci si può chiedere se non sia l'habitat stesso a chiedere una maggiore specializzazione delle indicazioni spaziali ${ }^{29}$.

Faut-il penser que le besoin de préciser, même de manière redondante, les concepts spatiaux est dû au style essentiellement oral qui caractérisent ces parlers? Comme pour les parlers romanches et alémaniques, on pourrait avancer l'hypothèse que l'abondance des constructions avec des adverbes et des prépositions est liée plus à l'oralité qui les caractérise qu'à l'influence des langues voisines ${ }^{30}$. Ces questions passionnantes restent ouvertes et mériteraient évidemment des études approfondies.

Par ailleurs, la transmission du patrimoine immatériel témoigne de l'ancrage très fort à l'espace géographique et anthropique, de l'appropriation de l'espace par ses habitants, de leurs exigences d'y faire référence. D'autre part, c'est grâce à ces récits que continue la transmission d'un savoir lié aussi bien à la culture matérielle qu’immatérielle.

Federica DiÉmoz

Université de Neuchâtel

28. Ibid., p. 35.

29. Ibid., p. 33.

30. R. Berthele, "Contact de langues et conceptualisations spatiales", p. 70. 


\section{BIBLIOGRAPHIE}

Aвry, Christian, "La légende des feuilles changées en or à Sixt (HauteSavoie) ", Le monde Alpin et Rhodanien, 2/2-4 (1974), p. 73-85.

Berthele, Raphaël, "Contact de langues et conceptualisations spatiales. Aspects de la sémantique et de la grammaire de la référence spatiale en sursilvan, vallader et surmiran", Vox Romanica, 66 (2007), p. 60-71.

Bourdin, Alexandre, Hérémence: son passé et notes sur le Val d'Hérens, Sion, Ed. du Château, 1973.

Bouvier, Jean-Claude (dir.), Nommer l'espace. Le monde Alpin et Rhodanien, 25/2-4, 1997.

Cardona, Giorgio Raimondo, I sei lati del mondo, Bari, Laterza, 1985.

CÉrésole, Alfred, Légendes des Alpes vaudoises, Lausanne, A. Imer, 1885.

Correvon, H., "La Fée de Cleibe», Archives des Traditions Populaires, 3 (1899), p. 142-145.

Diémoz, Federica, Pannatier, Gisèle, "Richesse et variété des patois autour des Alpes", Nouvelles du centre d'études francoprovençales René Willien, 45 (2002), p. 5-38.

FABre, Paul, "Ce que la toponymie peut apporter à la ... toponymie», Le Monde alpin et Rhodanien, 25/2-4 (1997), p. 13-20.

Gauchat, Louis, Jeanjaquet, Jules, Tappolet, Ernest, Tableaux phonétiques des patois suisses romands, Neuchâtel, Imprimerie Paul Attinger, 1925.

GPSR = GAUCHAT, Louis et al., Glossaire des patois de la Suisse romande, Neuchâtel, Imprimerie Paul Attinger, 1924-.

IsLER, Gotthilf, "Or ou charbon? A propos du symbolisme de quelques légendes des Alpes", in Les Suisses: modes de vie, traditions, mentalité, éd. par P. Hugger, Lausanne, Payot, 1992, t. 3, p. 1295-1306.

Jeanjaquet, Jules, «La fée de Premploz. Conte populaire en patois de Conthey (Valais)", Bulletin du GPSR, 7 (1908), p. 22-23.

—, "La peste à Nendaz», Bulletin du GPSR, 7 (1908), p. 46-50. 
Kristol, Andres et al., Dictionnaire toponymique des communes suisses/ Lexikon der schweizerischen Gemeindenamen/Dizionario toponomastico dei comuni svizzeri, Frauenfeld/Lausanne, Huber/Payot, 2005.

Lavallaz, Léon de, Essai sur le patois d'Hérémence (Valais-Suisse). Phonologie, morphologie, syntaxe, folklore, textes et glossaire, Paris, Librairie Droz, 1935 (1899).

Lévi-Strauss, Claude, La pensée sauvage, Paris, Plon, 1962.

Luyet, Basile, "Légendes de Savièse: recueil de contes valaisans publiés dans leur texte original en dialecte de Savièse avec une traduction française", Archives Suisses des Traditions populaires, 25 (1924), p. $20-46$.

MaIR, Walter N., «Transferenz oder autonome Bildung? Bemerkungen zum Problem der Partikelverben im Ladinischen, Friulanischen, Italienischen und Französischen", Zeitschrift für romanische Philologie, 100 (1984), p. 408-432.

Maître, Raphaël, Pannatier, Gisèle, "Graphie commune pour les patois valaisans", L'Ami du Patois, 36/143 (2009), p. 93-103.

Montelle, Edith, Waldmann, Richard, Brüsch, Béat (ill.), Les plus beaux contes de Suisse, Lausanne, Ed. Mondo, 1987.

Muret, Ernest, "Adverbes préposés à un complément de lieu dans les patois valaisans", in Festschrift Louis Gauchat, Aarau, H.R. Sauerländer, 1926, p. 79-94.

Murphy, William P., «Oral Literature», Annual Review of Anthropology, 7 (1978), p. 113-136.

Schüle, Rose-Claire, Inventaire lexicologique du parler de Nendaz (Valais), Berne, Francke, 3 vols, 1963, 1998, 2006.

-, Les vouivres dans le ciel de Nendaz. Ethnographie du ciel et des astres, du temps, de la terre, des plantes et des animaux réels et fabuleux à Nendaz, Baden, Hier+Jetzt, 2011.

Segaud, Marion, Anthropologie de l'espace: habiter, fonder, distribuer, Paris, A. Colin, 2007.

Simonsen, Michèle, Le conte populaire français, Paris, PUF, 1981.

Sornicola, Rosanna, "Per una tipologia del parlato nelle lingue romanze: il caso dei pronomi soggetto ", in Variation linguistique et enseignement des langues. Langue parlée, langue écrite, éd. par L. Rabassa, M. Roché, CERCLID, 9 (1997), p. 53-71. 
Tuaillon, Gaston, "Le francoprovençal: progrès d'une définition", Travaux de linguistique et de littérature, 10 (1972), p. 293-339.

—, "Le francoprovençal s'explique-t-il par les Burgondes?», Nouvelles du Centre d'études francoprovençales René Willien, 47 (2003), p. 104113.

Crédits photographiques

Pl. I.1 :

D’après G. Tuaillon, «Le francoprovençal».

Fig. 1:

Coll. privée de Madame Rose-Claire Schüle.

Fig. 2:

Coll. privée de Madame Rose-Claire Schüle. 
